\title{
An Improved Monkey Algorithm for a 0-1 Knapsack Problem
}

\author{
Yongquan Zhou ${ }^{1} \quad$ Xin Chen ${ }^{1,2} \quad$ Guo Zhou $^{3}$ \\ ${ }^{1}$ College of Information Science and Engineering, \\ Guangxi University for Nationalities, Nanning, Guangxi, 530006, China \\ ${ }^{2}$ School of software, Dalian University of Technology, Dalian 116024, China \\ ${ }^{3}$ Institute of Computing Technology, Chinese Academy of Sciences, Beijing, 100081, China \\ e-mail: yongquanzhoul126. com
}

\begin{abstract}
The 0-1 knapsack problem is a classic combinational optimization problem. However, many exiting algorithms have low precision and easily fall into local optimal solutions to solve the $0-1$ knapsack problem. In order to overcome these problems, this paper proposes a binary version of the monkey algorithm where the greedy algorithm is used to strengthen the local search ability, the somersault process is modified to avoid falling into local optimal solutions, and the cooperation process is adopted to speed up the convergence rate of the algorithm. To validate the efficiency of the proposed algorithm, experiments are carried out with various data instances of 0-1 knapsack problems and the results are compared with those of five metaheuristic algorithms.
\end{abstract}

Keywords: monkey algorithm; binary version of the monkey algorithm; knapsack problem; cooperation process; greedy strategy.

\section{Introduction}

Knapsack problems (KPs) were first proposed by Dantzing in the 1950s [1] and subsequently attracted many scholars. KPs appear in real-world decision-making processes in a wide variety of fields, for example, finding the least wasteful way to cut raw materials, selection of capital investments and financial portfolios, selection of assets for asset-backed securitization, resource allocation, cargo loading, etc. Thus far, people have put forward many effective algorithms based on exact algorithms and heuristic algorithms for solving knapsack problems.

Zou et al described a novel global harmony search (HS) algorithm that includes two important operations, position updating and genetic mutation, for solving 0-1 knapsack problems [2]. The experiment shows the proposed algorithm has good performance for solving large-scale knapsack problems. Xiang et al also considered a novel global-best harmony search algorithm that combines a two-phase repair operator to repair an infeasible harmony vector and to further improve a feasible solution [3]. Changdar et al considered an improved ant colony optimization (ACO) approach to solve 0-1 knapsack problems in a fuzzy environment [4]. The proposed algorithm creates $n$ candidate groups for $n$ objects; each ant selects a candidate value from each group. Bansal et al proposed a modified particle swarm optimization called MBPSO based on binary particle swarm optimization (BPSO) [5] for solving 0-1 knapsack problems and multidimensional knapsack problems [6]. Azad et al proposed a simplified binary version of the artificial fish swarm algorithm (AFSA) for solving 0-1 quadratic knapsack problems [7]; the random heuristic drop_item procedure is used to make the points feasible, and the heuristic add_item is also implemented to improve the quality of the solutions. Glover used an improved greedy algorithm and surrogate constraints for linear and quadratic knapsack problems [8]. Sitarz studied the relations between multiple criteria dynamic programming (MCDP) and the multiple knapsack problem[9]; the paper also showed how to use MCDP methods to solve multiple knapsack 
problems. Gao et al presented a novel quantum-inspired artificial immune system called MOQAIS, which is composed of a quantum-inspired artificial immune algorithm (QAIS) and an artificial immune system (BAIS) for multiobjective knapsack problems [10]. QAIS is responsible for exploration of the search space, and BAIS is applied for exploitation of the search space. García-Martínez proposed a tabu-enhanced destruction mechanism for an iterated greedy search in studying quadratic multiple knapsack problems [11]; the method records the last removed objects and avoids removing them again in subsequent iterations. Baykasoğlu et al used a priority-based encoding technique for a firefly algorithm (FA) to construct feasible solutions and prevent infeasibility for solving dynamic multidimensional knapsack problems [12]. Hifi et al used a dichotomous search-based exact method based on decomposing the original problem into a series of knapsack problems and introduced new upper bounds and incremental valid lower bounds in the interval search [13]. Levin et al studied the stochastic behavior of the knapsack problem and defined a variant problem in which item values are deterministic and item sizes are independent random variables [14]. Zhao used a nonlinear reductive dimension approximate algorithm for the knapsack problem [15]. Liu et al removed useless solution regions before applying simulated annealing (SA) to solve a knapsack problem and extracted the most possible part of the optimal solution space from the whole optimal solution space [16]. Saraç et al used a genetic algorithm (GA) and a hybrid solution approach for solving a developed model called the generalized quadratic multiple knapsack problems (G-QMKP) [17]. The experiment shows the proposed hybrid solution approach can obtain good solutions in a reasonable time for a large-scale problem. Lin investigated using GA in solving the fuzzy knapsack problem [18]; this method simulates a fuzzy number by distributing it into partition points and uses GA to evolve the values in each partition point.

The monkey algorithm (MA) is a new type of swarm intelligence based algorithm. It was proposed by Zhao and Tang in 2008 and is derived from simulation of the mountain-climbing processes of monkeys [19]. It consists of three processes: the climb process, watch-jump process and somersault process. The climb process is designed to gradually improve the objective function value. However, MA will spend considerable computing time searching for local optimal solutions in the climb process. To reduce the computing time and speed up the convergence rate, Chen and Zhou introduced the inertial step in the climb process and combined the simple method after the somersault process [20]. The watch-jump process can speed up the convergence rate of the algorithm, the purpose of the somersault process is to make monkeys find new search domains to avoid falling into local search. The algorithm has the advantages of simple structure, strong robustness, and not easy falling into local optimal solutions. Therefore, MA has been successfully applied in solutions to various optimization problems, such as transmission network expansion planning [21], intrusion detection technology [22], optimal sensor placement in structural health monitoring [23], the optimization of gas filling station project scheduling problem [24], the clustering analysis problem [25], etc. In this paper, the 0-1 knapsack problem will be studied, and a binary version of the monkey algorithm which combines cooperation process and greedy strategy (CGMA) is proposed. The algorithm improves the calculation accuracy and increases the convergence speed of the algorithm to a certain degree. The numerical experiment results show that the proposed algorithm has good performance in solving the 0-1 knapsack problem. It can be an efficient alternative for solving the 0-1 knapsack problem.

\section{The 0-1 Knapsack Problem}

The 0-1 knapsack problem is a typical NP-hard problem in operations research. The problem is defined 
as follows:

Given a set of items $O=\left\{o_{1}, o_{2}, \cdots, o_{n}\right\}$, each with a weight $w_{i}$ and a value $p_{i}$, determine the number of each item to include in a collection so that the total weight $W X$ is less than or equal to a given limit and the total value $P X \quad$ is as large as possible. It derives its name from the problem faced by someone who is constrained by a fixed-size knapsack and must fill it with the most valuable items. Its mathematical model is as follows:

$$
\left\{\begin{array}{l}
\max \text { imize } f\left(x_{1}, x_{2}, \cdots, x_{n}\right)=P X=\sum_{i=1}^{n} p_{i} x_{i} \\
\text { subject to } W X=\sum_{i=1}^{n} w_{i} x_{i} \leq V
\end{array}\right.
$$

and $x_{j} \in\{0,1\}, j=1,2, \cdots, n$. Where $P=\left(p_{1}, p_{2}, \cdots, p_{n}\right), W=\left(w_{1}, w_{2}, \cdots, w_{n}\right)$ represent the value vector and weight vector of all items. $V$ is the maximum capacity of the knapsack. $x_{i}=1$ indicates that item $i$ is included in the knapsack and $x_{i}=0$ that it is not.

\section{Description of Modified Monkey Algorithm}

The monkey algorithm was first proposed to solve numerical optimization problems as a new swarm intelligence based algorithm stemmed from the mountain-climbing behavior of monkeys [19]. Assume that there are many mountains in a given field. At the beginning, the monkeys climb up from their respective positions to find the mountaintops (this action is called climb process). When a monkey get the top of its mountain, it will find a higher mountain within the sight and jump somewhere of the mountain from the current position (this action is called watch-jump process), then repeat the climb process. After repetitions of the climb process and the watch-jump process, each monkey will somersault to a new search domain to find a much higher mountaintop (this action is called somersault process).

This paper proposed a binary version of the monkey algorithm where the greedy algorithm is used to correct the infeasible solutions and to improve the quality of the feasibility, the somersault process is modified to avoid falling into local search, the cooperation process is implemented to speed up the convergence rate, and the control parameter is used to keep the population diversity. The algorithm consists of 5 parts, the climb process, watch-jump process, greedy strategy repair process, cooperation process and somersault process.

\subsection{Coding method}

For the 0-1 knapsack problem, each item has two different status, namely the item has been included in the knapsack or it remains out. First, $M$ is defined as the population size of monkeys. For monkey $i$, its position is denoted as a vector $X_{i}=\left(x_{i 1}, x_{i 2}, \cdots, x_{i n}\right)$, and this position will be employed to express a solution of the 0-1 knapsack problem, where $x_{i j} \in\{0,1\}$ and $j=1,2, \cdots, n, n$ is the number of the items. $x_{i j}=1$ indicates the item $j$ is included in the knapsack and $x_{i j}=0$ indicates it is not. 


\subsection{Initial population}

In CGMA, the initial population is randomly generated. The random initialization process of $M$ monkeys and $n$ items is as follows:

for $i=1$ to $M$ do
for $j=1$ to $N$ do
$x[i][j]=\operatorname{rand}() ;$
If $x[i][j]<0.5$
$x[i][j]=0 ;$
else
$x[i][j]=1 ;$
endif
endfor
endfor

where $x_{i, j}$ represents the $j$ th component in the vector $X_{i}$.

\subsection{Climb Process}

According to the idea of pseudo-gradient-based simultaneous perturbation stochastic approximation (SPSA) [27], the climb process is a step-by-step procedure to improve the objective function by choosing a better one between two positions that are generated around the current position. For the monkey $i$, its position is $X_{i}=\left(x_{i 1}, x_{i 2}, \cdots, x_{i n}\right), i=1,2, \cdots, M$, respectively. $f\left(X_{i}\right)$ is the corresponding objective function value. The improved climb process is given as follows:

(1) Randomly generate two vectors $\Delta x_{i}^{\prime}=\left(\Delta x_{i 1}^{\prime}, \Delta x_{i 2}^{\prime}, \cdots, \Delta x_{i n}^{\prime}\right)$ and $\Delta x_{i}^{\prime \prime}=\left(\Delta x_{i 1}^{\prime \prime}, \Delta x_{i 2}^{\prime \prime}, \cdots, \Delta x_{i n}^{\prime \prime}\right)$, where

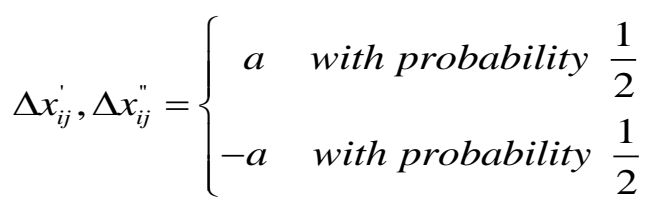

$j=1,2, \cdots, n$, respectively. The parameter $a(a>0)$, called the step of the climb process, can be determined by specific situations. Here, set the climb step $a=1$ for the $0-1$ knapsack problem.

(2) Set $x_{i j}^{\prime}=\left|x_{i j}-\Delta x_{i j}^{\prime}\right|$ and $x_{i j}^{\prime \prime}=\left|x_{i j}-\Delta x_{i j}^{\prime \prime}\right|, j=1,2, \cdots, n$, respectively, where $|x|$ represents the absolute value of $x$. Set $X_{i}^{\prime}=\left(x_{i 1}^{\prime}, x_{i 2}^{\prime}, \cdots, x_{i n}^{\prime}\right), X_{i}^{\prime \prime}=\left(x_{i 1}^{\prime \prime}, x_{i 2}^{\prime \prime}, \cdots, x_{i n}^{\prime \prime}\right)$.

(3) Calculate $f\left(X_{i}^{\prime}\right)$ and $f\left(X_{i}^{\prime \prime}\right), i=1,2, \cdots, M$, respectively.

(4) If $f\left(X_{i}^{\prime}\right)>f\left(X_{i}^{\prime \prime}\right)$ and $f\left(X_{i}^{\prime}\right)>f\left(X_{i}\right)$, set $X_{i}=X_{i}^{\prime}$. If $f\left(X_{i}^{\prime \prime}\right)>f\left(X_{i}^{\prime}\right)$ and $f\left(X_{i}^{\prime \prime}\right)>f\left(X_{i}\right), X_{i}=X_{i}^{\prime \prime}$.

(5) Repeat steps (1) to (4) until the maximum allowable number of iterations (called the climb 
number, denoted by $N c$ ) has been reached.

\subsection{Watch-jump Process}

When the monkeys reach the top of the mountains, each monkey will take a look and determine whether there are higher points than the current one within its sight. If yes, it will jump somewhere of the mountain from the current position and then repeat the climb process. For the monkey $i$, its position is $X_{i}=\left(x_{i 1}, x_{i 2}, \cdots, x_{i n}\right), i=1,2, \cdots, M$. The improved watch-jump process is given as follows:

(1) Randomly generate a real $y_{j}$ in interval $\left[x_{i j}-b, x_{i j}+b\right], j=1,2, \cdots, n$, respectively, where $b$ eyesight of the monkey can be determined by specific situations. Set $Y=\left(y_{1}, y_{2}, \cdots, y_{n}\right)$.

(2) Because of the real $y_{j} \in[-1,2]$, if $y_{j}<0.5$, set $y_{j}=0$; otherwise, set $y_{j}=1$.

(3) Calculate $f(Y), i=1,2, \cdots, M$, respectively.

(4) If $f(Y)>f\left(X_{i}\right)$, then $X_{i}=Y$.

(5) Repeat the climb process steps (1) to (4) until the maximum allowable number of iterations (called the watch-jump number, denoted by $N w$ ) has been reached.

\subsection{Greedy Strategy}

An abnormal encode individual (who does not meet the constraint conditions) may be obtained in solving the 0-1 knapsack problem using the metaheuristic algorithms. In [28], the local search strategy-greedy algorithm (GTA) is introduced to correct the infeasible solutions and to improve the quality of the feasibility. Assume that $P=\left(p_{1}, p_{2}, \cdots, p_{n}\right)$ and $W=\left(w_{1}, w_{2}, \cdots, w_{n}\right)$ represent the value vector and weight vector of all items. $V$ is the maximum capacity of the knapsack. $X=\left(x_{1}, x_{2}, \cdots, x_{n}\right)$ is an abnormal encode individual, and $Y=\left(y_{1}, y_{2}, \cdots, y_{n}\right)$ is the adjusted normal solution. GTA is described as follows according to the 0-1 knapsack problem:

Let $Q\left(q_{1}, q_{2}, \cdots, q_{n}\right)$ be a sequence that sorts by value density $p_{i} / w_{i}$ from large to small for all items $x_{i}=1$ in the vector $X=\left(x_{1}, x_{2}, \cdots, x_{n}\right) . G T A(X, W, P, V)$ Algorithm [28]:

$k=1 ;$
Temp $=W_{Q[k]} ;$
while Temp $\leq V$ do
$y_{Q[k]}=1 ;$
$k=k+1 ;$
Temp $=$ Temp $+W_{Q[k]} ;$
endwhile
for $j=k$ to $n$ do
$y_{Q[j]}=0 ;$
endfor




\subsection{Cooperation Process}

After the climb process and the watch-jump process, each monkey will arrive at the top mountain in its neighborhood. However, they differ among all the monkeys. The purpose of the cooperation process is to make the monkeys find a better solution by cooperating with the monkey that has the best position. The monkeys will go forward along the direction of the best monkey. This process can speed up the convergence rate. Assume that the optimal position is $X^{*}=\left(x_{1}^{*}, x_{2}^{*}, \cdots, x_{n}^{*}\right)$. For the monkey $i$, its position is $X_{i}=\left(x_{i 1}, x_{i 2}, \cdots, x_{i n}\right), i=1,2, \cdots, M$. The cooperation process is given as follows:

(1) Randomly generate a real number $\alpha$ from the interval [0,1].

(2) If $\alpha<0.5$, then $y_{j}=x_{j}$, otherwise, $y_{j}=x_{j}^{*}, j=1,2, \cdots, n$, respectively.

(3) Update the monkeys' position $X_{i}$ with $Y$.

\subsection{Somersault Process}

Monkeys will reach the maximal mountaintops around their initial points after repetitions of the climb process and the watch-jump process, respectively. To find a higher mountaintop, each monkey will somersault to a new search domain. The new position is not blind to choose, but limited within a certain region, which is determined by the pivot and the somersault interval. Figure 1 shows the somersault process of the original MA [8]. The points $A, B, C, D$ represent monkeys. The point $P$ is the center of all monkeys, the somersault interval $[c, d]=[-1,1]$. For example, the monkey $A$ can reach any point (such as point $P, A 1, A 2$ ) within the circle $r 1$ with the somersault interval $[c, d]=[-1,1]$.

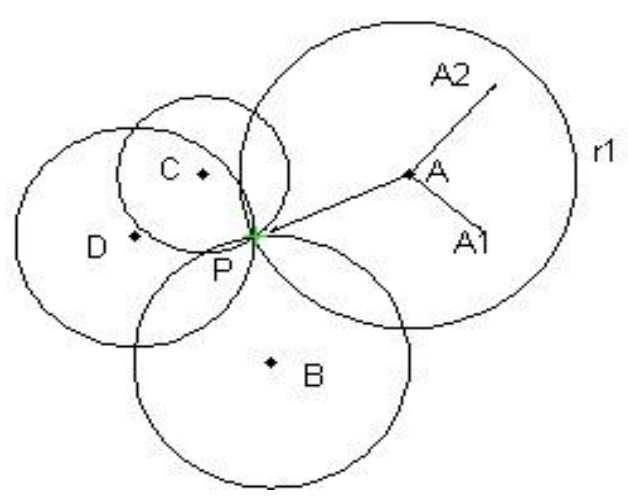

Figure 1. The somersault process of the original MA.

The somersault process can effectively prevent monkeys falling into the local search. However, after many iterations, the somersault process may lose efficacy. The monkeys will fall into the local optima domain, and the population diversity will decrease. In the original MA, the monkeys will somersault along the direction pointing to the pivot, which is equal to the barycenter of all monkeys' current positions. Here, we randomly choose a monkey's position as the pivot to replace the center of all monkeys and adopt a new somersault process. For the monkey $i$, its position is $X_{i}=\left(x_{i 1}, x_{i 2}, \cdots, x_{i n}\right), i=1,2, \cdots, M$. The improved somersault process is given as follows:

(1) Randomly generate real numbers $\theta$ from the interval $[c, d]$ (called the somersault interval, 
which governs the maximum distance that monkeys can somersault).

(2) Randomly generate an integer $k, k=1,2, \cdots, M$, respectively. Let the location of the monkey $k$ be the somersault pivot.

(3) Calculate

$$
y_{j}=x_{k j}+\theta\left(x_{k j}-x_{i j}\right)
$$

(4) Set $y_{j}=0$, if $y_{j}<0.5$; otherwise, set $y_{j}=1$. Update the monkey's position $X_{i}$ with $Y$.

After repetitions of the somersault process, monkeys may reach the same domain to make the somersault process lose efficacy. In case of this problem, we set a parameter called "limit" to control monkeys running into the local optima solution. If the global optimal solution is not improved by a predetermined number of trials, the monkeys are abandoned and then reinitialized.

\subsection{Termination Condition}

Following the climb process, watch-jump process, greedy method optimization process, cooperation process and somersault process, all monkeys are ready for their next actions. The condition for terminating CGMA iteration could either be when the optimal solution has been found or when a relatively large number of iterations have been reached. To summarize, the whole flowchart of the CGMA to find the optimal solution of the 0-1 Knapsack problem is shown in Figure 2. 


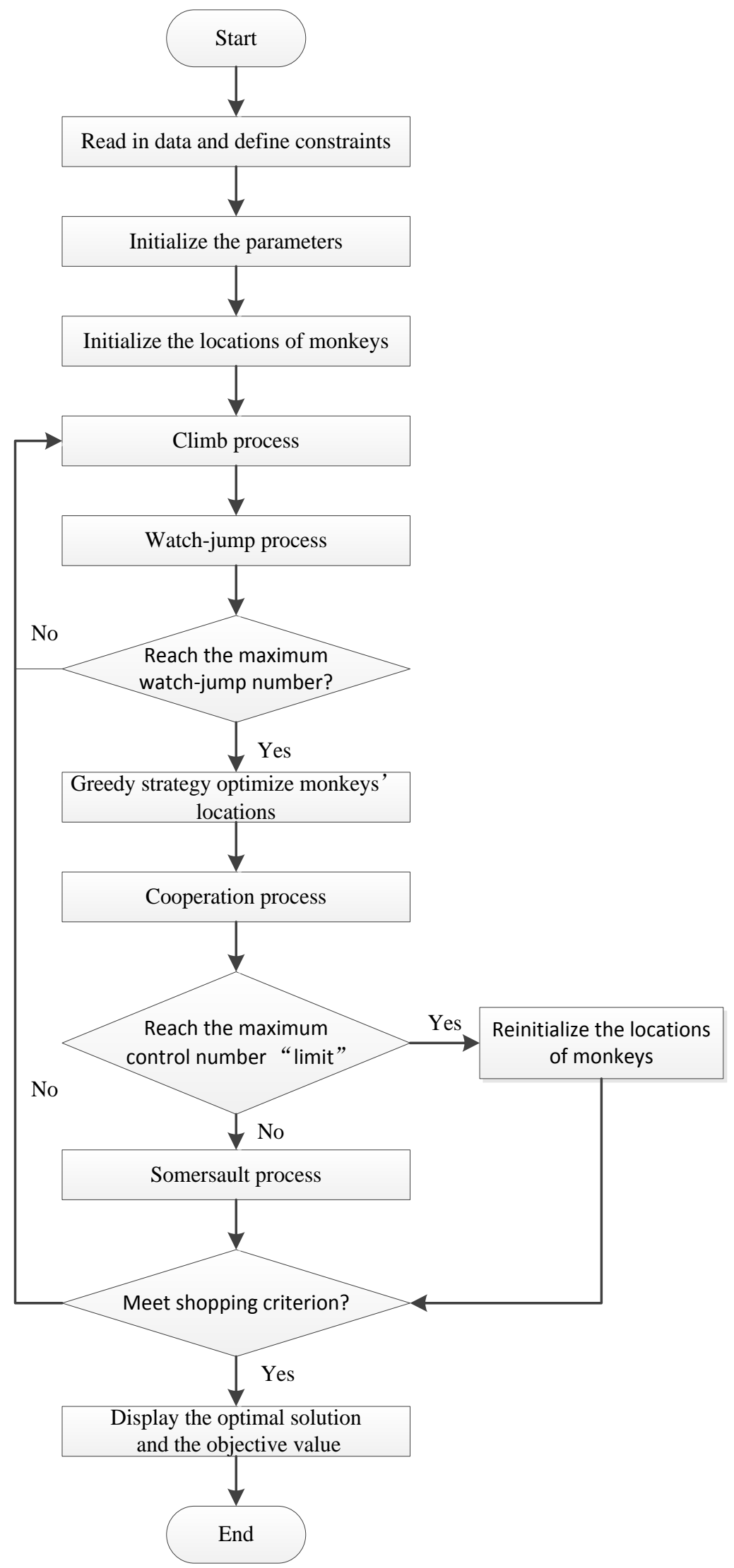

Figure 2. The flow chart of CGMA 


\section{Simulation Experiments}

In this section, the experiments were performed using a desktop computer with a $3.01 \mathrm{GHz}$ AMD Athlon(tm) II X4640 processor, 3GB of RAM, running a minimal installation of Windows XP. The application software was Matlab 2012a.

\subsection{Small-Scale Knapsack Problems}

To evaluate the performance of CGMA, it is compared with BPSO [6], MBPSO [6], NGHS [2], DGHS [3], and S-bAFSA [7]. To make a fair comparison, we use the maximum number of function evaluations (maxFEs) as the evaluation standard. In addition, the other specific parameters of the algorithms are given as follows:

For BPSO and MBPSO, the population size is set to 5. The acceleration coefficients are $c 1=2, c 2=2$. The maximum velocity $V_{\max }$ is set to 2 .

For NGHS, the harmony memory size HMS, namely, the population size, is set to 5; the mutation probability $p_{m}=2 / N$, and the penalty coefficient is set to $10^{20}$, which is the same as those utilized in [13].

For DGHS, HMS=5. The maximum and minimum harmony memory consideration rate $H M C R_{\max }$, $H M C R_{\min }$ are set to 0.95 and 0.3 , respectively. The pitch adjusting rate $P A R=0.75$.

For CGMA, the population size is 10 . The climb number $N c=2$, watch-jump number $N_{w}=2$, climb step $a=1$, eyesight $b=1$, and somersault interval $[c, d]=[-1,1]$.

Here, two sets of small-scale knapsack problems are selected to test the performance of CGMA. The first set that contains 10 instances is taken from [2]. The second set comes from [6] and is tested in [4] [29] also. The number of items in these instances range between 8 and 24, and the maxFEs are listed in the table 2. For every instance, each algorithm is run 50 times individually with random initial solutions.

Table 1. Results obtained by the algorithms for the first set

\begin{tabular}{|c|c|c|c|c|c|c|}
\hline Problem & The optimal value & Algorithm & The optimal solution vector & Best & Worst & Mean \\
\hline F1 & 295 & CGMA & 0111000111 & 295 & 295 & 295 \\
\hline F2 & 1024 & CGMA & 11111111111110101011 & 1024 & 1024 & 1024 \\
\hline F3 & 35 & CGMA & 1101 & 35 & 35 & 35 \\
\hline F4 & 23 & CGMA & 0101 & 23 & 23 & 23 \\
\hline F5 & 481.07 & CGMA & 001010110111011 & 481.07 & 481.07 & 481.07 \\
\hline F6 & 52 & CGMA & 0010111111 & 52 & 52 & 52 \\
\hline F7 & 107 & CGMA & 1001000 & 107 & 107 & 107 \\
\hline F8 & 9767 & CGMA & 11111111001000011000000 & 9767 & 9767 & 9767 \\
\hline F9 & 130 & CGMA & 11110 & 130 & 130 & 130 \\
\hline F10 & 1025 & CGMA & 11111111101111010111 & 1025 & 1025 & 1025 \\
\hline
\end{tabular}

Table 1 shows the results of CGMA for the first set. Here, the control parameter is set as $\lim i t=10$ (10 refers to iterations, and each iteration may need multiple function evaluations), the maxFEs is set to 1000 for all algorithms. As observed from the results, for the small-scale knapsack problems, CGMA can obtain the global optimal solution in all runs. Shi [32] proposed an improved ant colony algorithm to solve the knapsack problems (test problem 1 and 2), but the optimal solutions 
cannot be obtained in the paper. The optimal value of test problem 1 obtained by CGMA is 295, and the best value of test problem 2 is 1024. For the test problems F3, F4, and F5, the optimal solutions can be obtained by CGMA in all runs. For the test problem F6, Zou proposed a global harmony search algorithm to solve the problem in [2]. Unfortunately, the optimal solution of the problem cannot be found by NGHS [2]. The optimal solution for F6 obtained by CGMA is $x=(0,0,1,0,1,1,1,1,1,1)$, and the optimal value is 52 . For the test problem F7, F8, F9, F10, the optimal solution can be found by CGMA, HS, and NGHS in all runs. The optimal values of the test problem F7, F8, F9 and F10 are 107, 9767,130 and 1025, respectively.

Table 2. Results obtained by the algorithms for the second set

\begin{tabular}{|c|c|c|c|c|c|c|c|c|}
\hline Problem & Instance & & BPSO & MBPSO & NGHS & DGHS & S-bAFSA & CGMA \\
\hline \multirow{2}{*}{ F11 } & \multirow{2}{*}{ ks_8a } & Mean & 3921857.19 & 3924400 & 3924400 & 3924400 & 3924400 & 3924400 \\
\hline & & Best & 3924400 & 3924400 & 3924400 & 3924400 & 3924400 & 3924400 \\
\hline \multirow{2}{*}{ F12 } & \multirow{2}{*}{ ks_8b } & Mean & 3807911.86 & 3813669 & 3813669 & 3813669 & 3813669 & 3813669 \\
\hline & & Best & 3813669 & 3813669 & 3813669 & 3813669 & 3813669 & 3813669 \\
\hline \multirow{2}{*}{ F13 } & \multirow{2}{*}{ ks_8c } & Mean & 3328608.71 & 3347452 & 3347452 & 3347452 & 3347452 & 3347452 \\
\hline & & Best & 3347452 & 3347452 & 3347452 & 3347452 & 3347452 & 3347452 \\
\hline \multirow{2}{*}{ F14 } & \multirow{2}{*}{ ks_8d } & Mean & 4186088.27 & 4187707 & 4187707 & 4187707 & 4187707 & 4187707 \\
\hline & & Best & 4187707 & 4187707 & 4187707 & 4187707 & 4187707 & 4187707 \\
\hline \multirow{2}{*}{ F15 } & \multirow{2}{*}{ ks_8e } & Mean & 4932737.28 & 4954571.72 & 4955555 & 4955555 & 4955555 & 4955555 \\
\hline & & Best & 4955555 & 4955555 & 4955555 & 4955555 & 4955555 & 4955555 \\
\hline \multirow{2}{*}{ F16 } & \multirow{2}{*}{ ks_12a } & Mean & 5683694.29 & 5688552.41 & 5687724.44 & 5687460.74 & 5688498.02 & 5688887 \\
\hline & & Best & 5688887 & 5688887 & 5688887 & 5688887 & 5688887 & 5688887 \\
\hline \multirow{2}{*}{ F17 } & \multirow{2}{*}{ ks_12b } & Mean & 6478582.96 & 6493130.57 & 6486450.92 & 6498597 & 6498597 & 6498597 \\
\hline & & Best & 6498597 & 6498597 & 6498597 & 6498597 & 6498597 & 6498597 \\
\hline \multirow{2}{*}{ F18 } & \multirow{2}{*}{ ks_12c } & Mean & 5166957.08 & 5170493.3 & 5165079.86 & 5165079.80 & 5170626 & 5170626 \\
\hline & & Best & 5170626 & 5170626 & 5170626 & 5170626 & 5170626 & 5170626 \\
\hline \multirow{2}{*}{ F19 } & \multirow{2}{*}{ ks_12d } & Mean & 6989842.73 & 6992144.26 & 6991971.10 & 6992404 & 6992404 & 6992404 \\
\hline & & Best & 6992404 & 6992404 & 6992404 & 6992404 & 6992404 & 6992404 \\
\hline \multirow{2}{*}{ F20 } & \multirow{2}{*}{ ks_12e } & Mean & 5316879.59 & 5337472 & 5273974.06 & 5335555.92 & 5337472 & 5337472 \\
\hline & & Best & 5337472 & 5337472 & 5337472 & 5337472 & 5337472 & 5337472 \\
\hline \multirow{2}{*}{ F21 } & \multirow{2}{*}{ ks_16a } & Mean & 7834900.26 & 7843073.29 & 7795177.56 & 7850983 & 7850983 & 7850983 \\
\hline & & Best & 7850983 & 7850983 & 7850983 & 7850983 & 7850983 & 7850983 \\
\hline \multirow{2}{*}{$\mathrm{F} 22$} & \multirow{2}{*}{ ks_16b } & Mean & 9334408.62 & 9350353.39 & 9253153.36 & 9352998 & 9352998 & 9352998 \\
\hline & & Best & 9352998 & 9352998 & 9352998 & 9352998 & 9352998 & 9352998 \\
\hline \multirow{2}{*}{ F23 } & \multirow{2}{*}{ ks_16c } & Mean & 9118837.47 & 9144118.38 & 9055853.46 & 9151147 & 9151147 & 9151147 \\
\hline & & Best & 9151147 & 9151147 & 9151147 & 9151147 & 9151147 & 9151147 \\
\hline \multirow{2}{*}{ F24 } & \multirow{2}{*}{ ks_16d } & Mean & 9321705.87 & 9337915.64 & 9310913.74 & 9316797.66 & 9321195.66 & 9345229.60 \\
\hline & & Best & 9348889 & 9348889 & 9348889 & 9348889 & 9348889 & 9348889 \\
\hline \multirow{2}{*}{$\mathrm{F} 25$} & & Mean & 7758572.21 & 7764131.81 & 7738799.78 & 7748285.68 & 77613877.20 & 77679297.20 \\
\hline & & Best & 7769117 & 7769117 & 7769117 & 7769117 & 7769117 & 7769117 \\
\hline F26 & ks_20a & Mean & 10707360.91 & 10720314.03 & 10592304.82 & 10727049 & 10727049 & 10727049 \\
\hline
\end{tabular}




\begin{tabular}{|c|c|c|c|c|c|c|c|c|}
\hline & & Best & 10727049 & 10727049 & 10727049 & 10727049 & 10727049 & 10727049 \\
\hline \multirow{2}{*}{ F27 } & \multirow{2}{*}{ ks_20b } & Mean & 9791306.65 & 9805480.48 & 9728979.66 & 9818261 & 9818261 & 9818261 \\
\hline & & Best & 9818261 & 9818261 & 9818261 & 9818261 & 9818261 & 9818261 \\
\hline \multirow{2}{*}{ F28 } & \multirow{2}{*}{ ks_20c } & Mean & 10703423.34 & 10710947.05 & 10585114.14 & 10709663.64 & 10710831.94 & 10712553.74 \\
\hline & & Best & 10714023 & 10714023 & 10714023 & 10714023 & 10714023 & 10714023 \\
\hline \multirow{2}{*}{ F29 } & \multirow{2}{*}{ ks_20d } & Mean & 8910152.57 & 8923712.21 & 8859297.78 & 8916496.80 & 8917872.80 & 8918423.20 \\
\hline & & Best & 8929156 & 8929156 & 8929156 & 8929156 & 8929156 & 8929156 \\
\hline \multirow{2}{*}{ F30 } & \multirow{2}{*}{ ks_20e } & Mean & 9349546.98 & 9355930.35 & 9324698.84 & 9357518.34 & 9357331.86 & 9357922.38 \\
\hline & & Best & 9357969 & 9357969 & 9357969 & 9357969 & 9357969 & 9357969 \\
\hline \multirow{2}{*}{ F31 } & \multirow{2}{*}{ ks_24a } & Mean & 13510432.96 & 13532060.07 & 13508995.14 & 13527887.56 & 13524874.30 & 13538097.18 \\
\hline & & Best & 13549094 & 13549094 & 13549094 & 13549094 & 13549094 & 13549094 \\
\hline \multirow{2}{*}{ F32 } & \multirow{2}{*}{ ks_24b } & Mean & 12205346.16 & 12223442.61 & 12160953.58 & 12233713 & 12233713 & 12233713 \\
\hline & & Best & 12233713 & 12233713 & 12233713 & 12233713 & 12233713 & 12233713 \\
\hline \multirow{2}{*}{ F33 } & \multirow{2}{*}{ ks_24c } & Mean & 12427880.56 & 12443349.03 & 12424467.44 & 12448158.86 & 12448550.28 & 12448618.30 \\
\hline & & Best & 12448780 & 12448780 & 12448780 & 12448780 & 12448780 & 12448780 \\
\hline \multirow{2}{*}{ F34 } & \multirow{2}{*}{ ks_24d } & Mean & 11792064.76 & 11803712.38 & 11736314.98 & 11810261.56 & 11810893.24 & 11808614.90 \\
\hline & & Best & 11815315 & 11815315 & 11815315 & 11815315 & 11815315 & 11815315 \\
\hline \multirow{2}{*}{ F35 } & \multirow{2}{*}{ ks_24e } & Mean & 13922797.55 & 13932526.16 & 13827901.40 & 13940099 & 13940099 & 13940099 \\
\hline & & Best & 13940099 & 13940099 & 13940099 & 13940099 & 13940099 & 13940099 \\
\hline
\end{tabular}

Table 2 shows the results for all the algorithms for the second test set, which includes 25 test problems. "lim it" is the control parameter. It is set to 10 for the test problems 11 to 20 and 20 for the other problems. The value of maxFEs is set to 1000 for the test problems 11 to 20 and 5000 for the other problems. The results of BPSO and MBPSO are from [6]. For the test problems F11 to F23, F26, F27, F32 and F35, CGMA can obtain the global optimal values in all runs, and the success rate (SR) can reach $100 \%$. For other test problems, the mean values of CGMA are obviously better than BPSO, MBPSO, NGHS and DGHS. BPSO cannot find the global optimal value on all problems with $\mathrm{SR}=100 \%$. MBPSO can find the global optimal values in all runs on F11 to F14, F20. NGHS can also find the global optimal values on five test problems with $\mathrm{SR}=100 \%$, that is, $\mathrm{F} 11$ to $\mathrm{F} 15$. However, the mean values of NGHS on all the test problems are worse than that of MBPSO except F15. In addition, the mean values of MBPSO on all the test problems are better than that of BPSO. DGHS can find the global optimal values on 14 problems with $\mathrm{SR}=100 \%$, that is, F11 to F15, F17, F19, F21 to F23, F26, F27, F32, F35. BPSO and NGHS are inferior to MBPSO. For the test problems F11 to F15, F17 to F23, F26, F27, F32, F35, S-bAFSA can obtain the global optimal values in all runs, and the success rate (SR) can reach $100 \%$. Only the mean value of S-bAFSA on the test problem F34 is better than that of CGMA. Its performance is obviously better than BPSO, MBPSO, NGHS and DGHS. BPSO and NGHS are inferior to MBPSO and DGHS.

In this subsection, for the small-scale knapsack problems, CGMA has a higher success rate than other algorithms on all the test problems. The proposed algorithm has better performance than BPSO, MBPSO, NGHS, DGHS and S-bAFSA. 


\subsection{The Large-Scale Knapsack Problems}

\subsubsection{Experimental results}

To evaluate the performance of CGMA further, this subsection will test many large-scale problems. The details of the instances are given as follows:

The test problem F36 [30], F37 [28], F38 [28], F39 [3] include 50, 50, 80, 100 items, respectively. The corresponding maximum capacities of the knapsacks are 1000, 959, 1173, 6718, respectively.

Weakly correlated problems F40 to F44 [31]: the weights $w_{j}$ are random integers uniformly distributed in $[10,100]$, and the profits $p_{j}$ are random integers uniformly distributed in $\left[w_{j}-10, w_{j}+10\right]$.

Strongly correlated problems F45 to F49 [31]: the weights $w_{j}$ are random integers uniformly distributed in $[10,100]$, and the profits $p_{j}$ are set to $w_{j}+10$.

Multiple strongly correlated problems F50 to F54 [31]: the weights $w_{j}$ are randomly distributed in $[10,100]$. If the weight $w_{j}$ is divisible by 6 , we set the $p_{j}=w_{j}+30 ;$ otherwise, set it to $p_{j}$ $=w_{j}+20$.

Profit ceiling problems F55 to F59 [31]: the weights $w_{j}$ are randomly distributed in $[10,100]$, and the profits $p_{j}$ are set to $p_{j}=3\left|w_{j} / 3\right|$.

For the test problems F40 to F59, the maximum capacity of the knapsack is $V=0.75 \sum_{j=1}^{n} w_{j}$.

For every test problem, each algorithm is run 50 times individually with random initial solutions. For all the test problems, the maxFEs is set to 50000 .

Table 3. Results obtained by the algorithms for 50 different runs on the test problems F36 to F39

\begin{tabular}{|c|c|c|c|c|c|c|c|}
\hline Problem & Algorithm & Best & Worst & Mean & Median & Std & SR \\
\hline \multirow{7}{*}{ F36 } & BPSO & 3071 & 2886 & 3006.08 & 3007.50 & 36.3159 & $0 \%$ \\
\cline { 2 - 8 } & MBPSO & 3103 & 3039 & 3077.80 & 3080 & 15.2235 & $2 \%$ \\
\cline { 2 - 8 } & NGHS & 3098 & 2912 & 3057 & 3066 & 35.1167 & 0 \\
\cline { 2 - 8 } & DGHS & 3103 & 3103 & 3103 & 3103 & 0 & $100 \%$ \\
\cline { 2 - 8 } & S-bAFSA & 3103 & 3103 & 3103 & 3103 & 0 & $100 \%$ \\
\cline { 2 - 8 } & CGMA & 3103 & 3103 & 3103 & 3103 & 0 & $100 \%$ \\
\hline \multirow{6}{*}{ F37 } & BPSO & 4843 & 4475 & 4681.90 & 4697 & 89.1486 & $0 \%$ \\
\cline { 2 - 8 } & MBPSO & 4882 & 4773 & 4840.22 & 4846 & 28.3717 & $8 \%$ \\
\cline { 2 - 8 } & NGHS & 4860 & 4538 & 4747.16 & 4771.50 & 83.4301 & 0 \\
\cline { 2 - 8 } & DGHS & 4882 & 4882 & 4882 & 4882 & 0 & $100 \%$ \\
\cline { 2 - 8 } & S-bAFSA & 4882 & 4882 & 4882 & 4882 & 0 & $100 \%$ \\
\cline { 2 - 8 } & CGMA & 4882 & 4882 & 4882 & 4882 & 0 & $100 \%$ \\
\hline \multirow{7}{*}{ F38 } & BPSO & 5138 & 4700 & 4937.04 & 4951.50 & 108.4808 & $0 \%$ \\
\cline { 2 - 8 } & MBPSO & 5183 & 5052 & 5131.82 & 5137 & 33.7939 & $2 \%$ \\
\cline { 2 - 8 } & NGHS & 5143 & 4849 & 5017.46 & 5020 & 78.0657 & $0 \%$ \\
\cline { 2 - 8 } & DGHS & 5181 & 5178 & 5179.56 & 5181 & 1.5140 & $0 \%$ \\
\hline
\end{tabular}




\begin{tabular}{|c|c|c|c|c|c|c|c|}
\hline & S-bAFSA & 5178 & 5178 & 5178 & 5178 & 0 & $0 \%$ \\
\cline { 2 - 8 } & CGMA & 5183 & 5167 & 5180.22 & 5181 & 2.9643 & $36 \%$ \\
\hline \multirow{7}{*}{ F39 } & BPSO & 26325 & 25010 & 25815.78 & 25820.50 & 280.3619 & $0 \%$ \\
\cline { 2 - 8 } & MBPSO & 26547 & 25919 & 26337.88 & 26363 & 142.1435 & $0 \%$ \\
\cline { 2 - 8 } & NGHS & 26507 & 24830 & 26066.14 & 26316 & 510.3133 & $0 \%$ \\
\cline { 2 - 8 } & DGHS & 26559 & 26547 & 26558.28 & 26559 & 2.8788 & $94 \%$ \\
\cline { 2 - 8 } & S-bAFSA & 26559 & 26541 & 26547.48 & 26544 & 7.6030 & $28 \%$ \\
\cline { 2 - 8 } & CGMA & 26559 & 26559 & 26559 & 26559 & 0 & $100 \%$ \\
\hline
\end{tabular}

Four test problems are selected to test the performance of the six algorithms. The best value, the worst value, the mean value, the median value, the standard deviation and the success rates (SR) are recorded in table 3. As observed from the results, CGMA can easily find the optimal values with $\mathrm{SR}=100 \%$ in most test problems except F38. DGHS and S-bAFSA can find the optimal values in all runs for F36 and F37. However, they fail to search the global optimal solution for F38. Only CGMA and MBPSO can find the optimal value with $\mathrm{SR}=36 \%$ and $\mathrm{SR}=2 \%$, respectively. The mean values of NGHS, MBPSO and PSO are obviously inferior to CGMA, DGHS and S-bAFSA for all test problems. The SR of BPSO, MBPSO, NGHS, DGHS and S-bAFSA are 0\%, 0\%, 0\%, $94 \%$ and 28\%, respectively. We note that CGMA has the lowest standard deviation for all test problems. The performance of S-bAFSA and DGHS is better than that of BPSO, MBPSO and NGHS.

Table 4 shows the optimal solutions for CGMA for the four test problems, where $T V / T W$ represents the corresponding total value and weight of the optimum solution. It is clear to see the improvement in CGMA over BPSO, MBPSO, NGHS, DGHS and F-bAFSA in terms of the boxplot as shown in Figure 3 to 6. These boxplots show the difference in CGMA, F-bAFSA, DGHS, NGHS, MBPSO and BPSO regarding the results for 50 independent runs. The boxplots for CGMA have greater value and less height than those of MBPSO, BPSO and NGHS. This means that the optimal value, the worst value, the mean value and the median value of CGMA are more than those of MBPSO, BPSO and NGHS.

Table 4. The results of CGMA for four test problems

\begin{tabular}{|c|c|c|}
\hline Problem & The optimal solution vector & $T V / T W$ \\
\hline F36 & 11010101111011011011011111110100001010011000001000 & $3103 / 1000$ \\
\hline F37 & 11111011111001100101011000100000111000011111111111 & $4882 / 959$ \\
\hline F38 & 11111111111111111111111111110111010100100 & $5183 / 1170$ \\
\hline F39 & 010110001000000000001000010000000000000 & \\
\hline
\end{tabular}




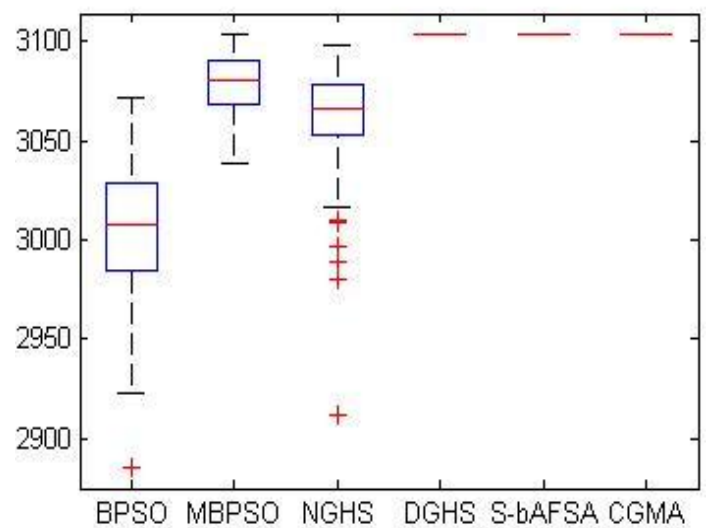

Figure 3. The boxplot of F36

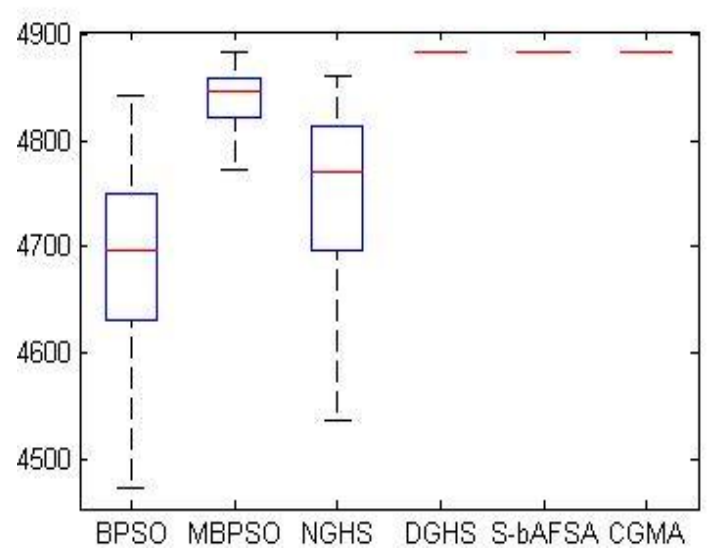

Figure 5. The boxplot of F38

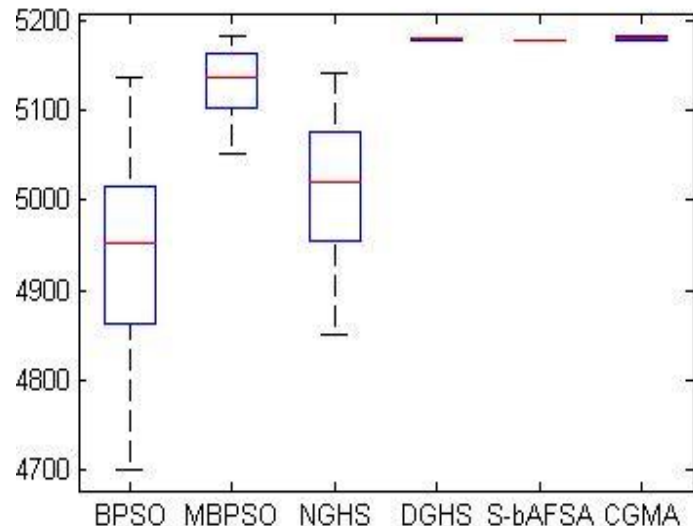

Figure 4. The boxplot of F37

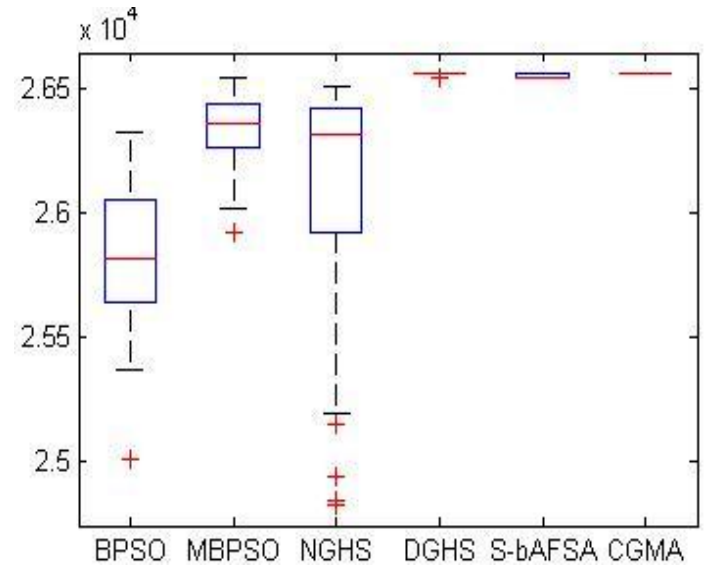

Figure 6. The boxplot of F39

Table 5. Results obtained by the algorithms for 50 different runs on weakly correlated problems

\begin{tabular}{|c|c|c|c|c|c|c|c|}
\hline Problem & Dim & Algorithm & Best & Worst & Mean & Median & Std \\
\hline \multirow{6}{*}{$\mathrm{F} 40$} & \multirow{6}{*}{200} & BPSO & 8671 & 8520 & 8600.74 & 8596.50 & 32.5713 \\
\hline & & MBPSO & 8757 & 8649 & 8701.70 & 8695.50 & 25.8727 \\
\hline & & NGHS & 8685 & 8486 & 8586.74 & 8588.50 & 32.8023 \\
\hline & & DGHS & 8822 & 8821 & 8821.70 & 8822 & 0.4629 \\
\hline & & S-bAFSA & 8822 & 8822 & 8822 & 8822 & 0 \\
\hline & & CGMA & 8823 & 8821 & 8822.18 & 8822 & 0.5226 \\
\hline \multirow{6}{*}{ F41 } & \multirow{6}{*}{300} & BPSO & 13201 & 13032 & 13126.12 & 13131.50 & 39.0429 \\
\hline & & MBPSO & 13314 & 13712 & 13246.84 & 13252.50 & 33.4520 \\
\hline & & NGHS & 13168 & 12963 & 13048.96 & 13055.50 & 39.7563 \\
\hline & & DGHS & 13489 & 13486 & 13488.34 & 13488 & 0.7722 \\
\hline & & S-bAFSA & 13489 & 13488 & 13488.98 & 13489 & 0.1414 \\
\hline & & CGMA & 13489 & 13489 & 13489 & 13489 & 0 \\
\hline \multirow{5}{*}{$\mathrm{F} 42$} & \multirow{5}{*}{500} & BPSO & 20488 & 20263 & 20359.98 & 20357.50 & 51.7555 \\
\hline & & MBPSO & 20601 & 20374 & 20486.92 & 20487 & 48.8934 \\
\hline & & NGHS & 20295 & 20001 & 20147.84 & 20156.50 & 63.6266 \\
\hline & & DGHS & 20961 & 20955 & 20958.64 & 20959 & 1.6383 \\
\hline & & S-bAFSA & 20961 & 20959 & 20959.84 & 20960 & 0.4677 \\
\hline
\end{tabular}




\begin{tabular}{|c|c|c|c|c|c|c|c|}
\hline & & CGMA & 20961 & 20960 & 20960.52 & 20961 & 0.5047 \\
\hline \multirow{6}{*}{$\mathrm{F} 43$} & \multirow{6}{*}{800} & BPSO & 33561 & 33217 & 33385.60 & 33376 & 74.8997 \\
\hline & & MBPSO & 33657 & 33328 & 33495.20 & 33490 & 67.2786 \\
\hline & & NGHS & 33133 & 32841 & 32983.30 & 32983 & 71.1854 \\
\hline & & DGHS & 34471 & 34447 & 34455.64 & 34455 & 4.5164 \\
\hline & & S-bAFSA & 34472 & 34442 & 34463.50 & 34465 & 8.3696 \\
\hline & & CGMA & 34472 & 34452 & 34463.96 & 34463.50 & 5.5841 \\
\hline \multirow{6}{*}{ F44 } & \multirow{6}{*}{1000} & BPSO & 40751 & 40445 & 40591.90 & 40583.50 & 77.9778 \\
\hline & & MBPSO & 40819 & 40527 & 40663.52 & 40667.50 & 80.1965 \\
\hline & & NGHS & 40214 & 39858 & 40024.58 & 40008 & 78.8388 \\
\hline & & DGHS & 41958 & 41916 & 41948.44 & 41948 & 6.7557 \\
\hline & & S-bAFSA & 41961 & 41944 & 41957.04 & 41961 & 5.2720 \\
\hline & & CGMA & 41961 & 41921 & 41950.56 & 41952.50 & 8.8275 \\
\hline
\end{tabular}

Table 6. Results obtained by the algorithms for 50 different runs on strongly correlated problems

\begin{tabular}{|c|c|c|c|c|c|c|c|}
\hline Problem & Dim & Algorithm & Best & Worst & Mean & Median & Std \\
\hline \multirow{6}{*}{ F45 } & \multirow{6}{*}{200} & BPSO & 10409 & 10319 & 10365.24 & 10367 & 21.7749 \\
\hline & & MBPSO & 10449 & 10359 & 10418.94 & 10419 & 19.2528 \\
\hline & & NGHS & 10429 & 10345 & 10389.94 & 10389 & 19.2613 \\
\hline & & DGHS & 10519 & 10519 & 10519 & 10519 & 0 \\
\hline & & S-bAFSA & 10519 & 10516 & 10518.74 & 10519 & 0.6328 \\
\hline & & CGMA & 10519 & 10519 & 10519 & 10519 & 0 \\
\hline \multirow{6}{*}{ F46 } & \multirow{6}{*}{300} & BPSO & 14420 & 14280 & 14338.42 & 14340 & 27.9614 \\
\hline & & MBPSO & 14469 & 14350 & 14399.54 & 14400 & 26.1879 \\
\hline & & NGHS & 14409 & 14270 & 14352.52 & 14359.50 & 33.9683 \\
\hline & & DGHS & 14589 & 14589 & 14589 & 14589 & 0 \\
\hline & & S-bAFSA & 14590 & 14587 & 14589.60 & 14590 & 0.8081 \\
\hline & & CGMA & 14589 & 14589 & 14589 & 14589 & 0 \\
\hline \multirow{6}{*}{ F47 } & \multirow{6}{*}{500} & BPSO & 24675 & 24504 & 24608.58 & 24605 & 41.7818 \\
\hline & & MBPSO & 24775 & 24625 & 24704.94 & 24705 & 38.96701 \\
\hline & & NGHS & 24695 & 24562 & 24631.46 & 24635 & 32.5013 \\
\hline & & DGHS & 25075 & 25055 & 25065 & 25065 & 6.3887 \\
\hline & & S-bAFSA & 25075 & 25070 & 25073.84 & 25074 & 1.2513 \\
\hline & & CGMA & 25075 & 25075 & 25075 & 25075 & 0 \\
\hline \multirow{6}{*}{ F48 } & \multirow{6}{*}{800} & BPSO & 37975 & 37745 & 37879.12 & 37885 & 51.8903 \\
\hline & & MBPSO & 38073 & 37834 & 37949.88 & 37953.50 & 49.6632 \\
\hline & & NGHS & 38045 & 37805 & 37916.82 & 37910 & 55.5866 \\
\hline & & DGHS & 38705 & 38685 & 38696.80 & 38695 & 8.0026 \\
\hline & & S-bAFSA & 38705 & 38705 & 38705 & 38705 & 0 \\
\hline & & CGMA & 38705 & 38685 & 38699.80 & 38705 & 7.8870 \\
\hline \multirow{3}{*}{ F49 } & \multirow{3}{*}{1000} & BPSO & 48822 & 48612 & 48712.76 & 48712 & 48.1692 \\
\hline & & MBPSO & 48872 & 48672 & 48764.12 & 48766.50 & 46.6820 \\
\hline & & NGHS & 48859 & 48600 & 48766.56 & 48772 & 59.6128 \\
\hline
\end{tabular}




\begin{tabular}{|c|c|c|c|c|c|c|c|}
\hline & DGHS & 49722 & 49692 & 49705.80 & 49702 & 8.0534 \\
\cline { 3 - 8 } & & S-bAFSA & 49731 & 49731 & 49731 & 49731 & 0 \\
\cline { 3 - 8 } & & CGMA & 49731 & 49711 & 49722.68 & 49721 & 6.4884 \\
\hline
\end{tabular}

Table 7. Results obtained by the algorithms for 50 different runs on multiple strongly correlated problems

\begin{tabular}{|c|c|c|c|c|c|c|c|}
\hline Problem & Dim & Algorithm & Best & Worst & Mean & Median & Std \\
\hline \multirow{6}{*}{ F50 } & \multirow{6}{*}{300} & BPSO & 19166 & 18766 & 18928.48 & 18924.50 & 88.4098 \\
\hline & & MBPSO & 19236 & 18876 & 19100.28 & 19114.50 & 73.8670 \\
\hline & & NGHS & 19076 & 18572 & 18803.18 & 18802.50 & 95.2834 \\
\hline & & DGHS & 19546 & 19536 & 19536.20 & 19536 & 14.1421 \\
\hline & & S-bAFSA & 19546 & 19546 & 19546 & 19546 & 0 \\
\hline & & CGMA & 19546 & 19546 & 19546 & 19546 & 0 \\
\hline \multirow{6}{*}{ F51 } & \multirow{6}{*}{500} & BPSO & 31823 & 31323 & 31633.98 & 31641 & 105.2533 \\
\hline & & MBPSO & 31973 & 31569 & 31772.14 & 31777.50 & 97.2997 \\
\hline & & NGHS & 31560 & 30920 & 31268.60 & 31280 & 141.4740 \\
\hline & & DGHS & 32713 & 32703 & 32712.80 & 32713 & 1.4142 \\
\hline & & S-bAFSA & 32723 & 32720 & 32720.78 & 32720 & 1.0554 \\
\hline & & CGMA & 32723 & 32723 & 32723 & 32723 & 0 \\
\hline \multirow{6}{*}{ F52 } & \multirow{6}{*}{800} & BPSO & 49652 & 49080 & 49373.62 & 49355 & 146.6236 \\
\hline & & MBPSO & 49643 & 48993 & 49339.66 & 49357.50 & 146.3074 \\
\hline & & NGHS & 49273 & 48227 & 48704.32 & 48701.50 & 205.2844 \\
\hline & & DGHS & 51312 & 51273 & 51290.28 & 51293 & 7.7276 \\
\hline & & S-bAFSA & 51312 & 51276 & 51309.50 & 51310 & 4.8582 \\
\hline & & CGMA & 51313 & 51273 & 51302.32 & 51313 & 12.9385 \\
\hline \multirow{6}{*}{ F53 } & \multirow{6}{*}{1000} & BPSO & 63341 & 62710 & 63073.92 & 63086 & 150.2352 \\
\hline & & MBPSO & 63241 & 62581 & 62964.92 & 62979 & 168.0327 \\
\hline & & NGHS & 62820 & 61991 & 62357.04 & 62367.50 & 185.9067 \\
\hline & & DGHS & 65391 & 65361 & 65376 & 65371 & 9.5298 \\
\hline & & S-bAFSA & 65391 & 65371 & 65384.22 & 65385.50 & 5.1992 \\
\hline & & CGMA & 65391 & 65361 & 65385 & 65391 & 8.8063 \\
\hline \multirow{6}{*}{ F54 } & \multirow{6}{*}{1200} & BPSO & 76240 & 75400 & 75715.82 & 75729 & 170.9516 \\
\hline & & MBPSO & 75981 & 74810 & 75409.46 & 75440 & 218.0730 \\
\hline & & NGHS & 75206 & 74350 & 74788.40 & 74781.50 & 192.9648 \\
\hline & & DGHS & 78721 & 78681 & 78703.40 & 78701 & 10.7968 \\
\hline & & S-bAFSA & 78721 & 78672 & 78716.36 & 78721 & 12.0995 \\
\hline & & CGMA & 78721 & 78671 & 78710.96 & 78721 & 19.5873 \\
\hline
\end{tabular}

Table 8. Results obtained by the algorithms for 50 different runs on profit ceiling problems

\begin{tabular}{|c|c|c|c|c|c|c|c|}
\hline Problem & Dim & Algorithm & Best & Worst & Mean & Median & Std \\
\hline \multirow{3}{*}{ F55 } & \multirow{3}{*}{300} & BPSO & 12717 & 12684 & 12700.26 & 12699 & 6.6142 \\
\cline { 3 - 8 } & & MBPSO & 12723 & 12693 & 12710.04 & 12711 & 6.3694 \\
\cline { 3 - 8 } & & NGHS & 12717 & 12687 & 12700.98 & 12699 & 6.4886 \\
\cline { 3 - 8 } & & DGHS & 12771 & 12771 & 12771 & 12771 & 0 \\
\hline
\end{tabular}




\begin{tabular}{|c|c|c|c|c|c|c|c|}
\hline & & S-bAFSA & 12771 & 12771 & 12771 & 12771 & 0 \\
\hline & & CGMA & 12771 & 12771 & 12771 & 12771 & 0 \\
\hline \multirow{6}{*}{ F56 } & \multirow{6}{*}{500} & BPSO & 20790 & 20748 & 20767.56 & 20766 & 9.4915 \\
\hline & & MBPSO & 20796 & 20763 & 20781.42 & 20781 & 7.4972 \\
\hline & & NGHS & 20790 & 20745 & 20769.12 & 20770.50 & 10.1411 \\
\hline & & DGHS & 20880 & 20880 & 20880 & 20880 & 0 \\
\hline & & S-bAFSA & 20880 & 20880 & 20880 & 20880 & 0 \\
\hline & & CGMA & 20880 & 20880 & 20880 & 20880 & 0 \\
\hline \multirow{6}{*}{ F57 } & \multirow{6}{*}{800} & BPSO & 33666 & 33618 & 33639.36 & 33639 & 9.7662 \\
\hline & & MBPSO & 33669 & 33621 & 33645.90 & 33645 & 11.3592 \\
\hline & & NGHS & 33669 & 33618 & 33643.98 & 33645 & 10.3142 \\
\hline & & DGHS & 33834 & 33831 & 33833.57 & 33834 & 1.0757 \\
\hline & & S-bAFSA & 33834 & 33834 & 33834 & 33834 & 0 \\
\hline & & CGMA & 33834 & 33831 & 33833.94 & 33834 & 0.4243 \\
\hline \multirow{6}{*}{ F58 } & \multirow{6}{*}{1000} & BPSO & 42345 & 42300 & 42324.12 & 42324 & 12.7993 \\
\hline & & MBPSO & 43339 & 42312 & 42359.06 & 42342 & 141.8400 \\
\hline & & NGHS & 42345 & 42294 & 42323.58 & 42324 & 11.1430 \\
\hline & & DGHS & 42561 & 42561 & 42561 & 42561 & 0 \\
\hline & & S-bAFSA & 42561 & 42561 & 42561 & 42561 & 0 \\
\hline & & CGMA & 42561 & 42561 & 42561 & 42561 & 0 \\
\hline \multirow{6}{*}{ F59 } & \multirow{6}{*}{1200} & BPSO & 49803 & 49743 & 49777.74 & 49777.50 & 14.9705 \\
\hline & & MBPSO & 49815 & 49761 & 49785.26 & 49785 & 13.5177 \\
\hline & & NGHS & 49812 & 49758 & 49790.76 & 49791 & 11.5132 \\
\hline & & DGHS & 50091 & 50082 & 50086.62 & 50088 & 2.9200 \\
\hline & & S-bAFSA & 50091 & 50091 & 50091 & 50091 & 0 \\
\hline & & CGMA & 50091 & 50079 & 50088.42 & 50088 & 2.9698 \\
\hline
\end{tabular}

For the test problems F40 to F59, the best value, the worst value, the mean value, the median value, and the standard deviation are recorded in table 5 to 8 .

For the weakly correlated problems, the mean values of CGMA are better than those of other algorithms except F44. CGMA can find the same global optimal value in 50 runs on the test problem F41. However, the standard deviation of DGHS is better than that of CGMA on the test problems F40, F43, F44. The mean values of CGMA are better than that of the other five algorithms except F44. The mean values of BPSO, MBPSO and DGHS are obviously inferior to that of CGMA, S-bAFSA and DGHS.

Table 6 records the results of the six algorithms on the strongly correlated problems. Because the profits $p_{j}$ are set to $w_{j}+10$, the profits are strongly correlated with the weights. CGMA can find the same global optimal values with Std=0 on the test problems F45, F46 and F47, and DGHS can also find the same global optimal values with $\mathrm{Std}=0$ on the test problems F45 and F46. However, the mean values of S-bAFSA are better than CGMA for F48 and F49.

For the multiple strongly correlated problems, CGMA can find the same global optimal values in all runs on the test problems F50 and F51. The mean values of CGMA are smaller than those of S-bAFSA for F52 and F54. 
The standard deviation of BPSO, MBPSO and NGHS are great, but CGMA, S-bAFSA and DGHS still have lower standard deviations on all the test problems.

Table 8 records the results of the five algorithms on the profit ceiling problems. The profits $p_{j}$ are set to $p_{j}=3\left|w_{j} / 3\right|$, that is, the profits have a strong relation with weights. CGMA and DGHS can find the same global values in all runs on the test problem F55, F56 and F58. S-bAFSA can easily find the optimal value in all test problems. All the algorithms have a small standard deviation on all the test problems. The mean value of S-bAFSA is obviously better than that of other algorithms.

CGMA has the best global exploration ability, and the mean values of CGMA are better than other algorithms on most test problems. The performance of DGHS is somewhat inferior to that of S-bAFSA. The global exploration ability of MBPSO is stronger than that of NGHS and BPSO.

\subsubsection{Algorithm Evaluation}

\section{1) Algorithm comparison}

For most swarm intelligence based algorithms, the maxFE impacts the precision of the solution. In this subsection, eight 0-1 knapsack problems of large size are devised to testify and compare the performance of CGMA, S-bAFSA, DGHS, NGHS, MBPSO and BPSO with different maxFEs. $N$ is set to $100,200,300,500,800,1000,1200$ and 1500, respectively, where $N$ represents the size of the test problem. For each $N$, the values of weight and profit are generated randomly: The weight $w_{j}(j=1,2, \cdots, N)$ is between 5 and 20 ; the profit $p_{j}(j=1,2, \cdots, N)$ is between 50 and 100 . For the eight values of $N$, the maximum capacity of knapsack $V$ is accordingly set to 1100, 1500, $1700,2000,5000,10000,14000$ and 16000, respectively. For each $N$, once the randomly generated parameters are determined, the same parameters are used to test the performance of three algorithms for 50 independent runs. The results obtained by six algorithms are presented in table 9 to 16 .

Table 9. The results of the test problems F60 with different maxFEs

\begin{tabular}{|c|c|c|c|c|c|c|}
\hline Algorithm & maxFEs & Best & Worst & Mean & Median & Std \\
\hline \multirow{3}{*}{ BPSO } & 10000 & 7481 & 7382 & 7452.86 & 7459 & 24.1475 \\
& 30000 & 7482 & 7322 & 7433.24 & 7448.50 & 42.4591 \\
& 50000 & 7478 & 7363 & 7439.42 & 7446 & 28.8883 \\
\hline \multirow{3}{*}{ MBPSO } & 10000 & 7482 & 7449 & 7474.94 & 7478 & 7.5442 \\
& 30000 & 7482 & 7430 & 7472.42 & 7476 & 10.9807 \\
& 50000 & 7482 & 7414 & 7473.50 & 7477.50 & 12.8575 \\
\hline \multirow{3}{*}{ NGHS } & 10000 & 7482 & 7480 & 7481.26 & 7481 & 0.4870 \\
& 30000 & 7482 & 7476 & 7481.16 & 7481 & 1.1669 \\
& 50000 & 7482 & 7476 & 7481.18 & 7481 & 1.4097 \\
\hline \multirow{3}{*}{ DGHS } & 10000 & 7480 & 7478 & 7478.50 & 7478 & 0.6776 \\
& 30000 & 7481 & 7476 & 7479.78 & 7480 & 0.6788 \\
& 50000 & 7481 & 7480 & 7480.84 & 7481 & 0.3703 \\
\hline S-bAFSA & 10000 & 7480 & 7474 & 7475.64 & 7475 & 2.1643 \\
\hline
\end{tabular}




\begin{tabular}{|c|c|c|c|c|cc|c|}
\hline & 30000 & 7480 & 7274 & 7476.18 & 7475 & 2.2010 \\
& 50000 & 7480 & 7474 & 7475.36 & 7475 & 1.7930 \\
\hline \multirow{3}{*}{ CGMA } & 10000 & 7481 & 7480 & 7480.44 & 7480 & 0.5014 \\
& 30000 & 7481 & 7480 & 7480.84 & 7481 & 0.3703 \\
& 50000 & 7481 & 7481 & 7481 & 7481 & 0 \\
\hline
\end{tabular}

Table 10. The results of the test problems F61 with different maxFEs

\begin{tabular}{|c|c|c|c|c|c|c|}
\hline Algorithm & maxFEs & Best & Worst & Mean & Median & Std \\
\hline \multirow{3}{*}{ BPSO } & 10000 & 11057 & 10455 & 10811.96 & 10794.50 & 127.1729 \\
& 30000 & 11090 & 10434 & 10829.02 & 10820.55 & 129.5015 \\
& 50000 & 11276 & 10935 & 11121.68 & 11113 & 70.9019 \\
\hline \multirow{3}{*}{ MBPSO } & 10000 & 11287 & 10900 & 11106.62 & 11112.50 & 89.7804 \\
& 30000 & 11331 & 10916 & 11123.60 & 11120 & 92.0459 \\
& 50000 & 11429 & 11140 & 11307.08 & 11308 & 63.78242 \\
\hline \multirow{3}{*}{ NGHS } & 10000 & 10570 & 9472 & 10062.98 & 10066 & 194.2577 \\
& 30000 & 10535 & 9919 & 10211.36 & 10190.50 & 166.0890 \\
& 50000 & 10673 & 9756 & 10256.52 & 10283 & 188.2011 \\
\hline \multirow{3}{*}{ DGHS } & 10000 & 11537 & 11529 & 11528.38 & 11529 & 4.6418 \\
& 30000 & 11537 & 11532 & 11534.98 & 11535 & 1.9005 \\
& 50000 & 11543 & 11537 & 11540.04 & 11540 & 2.2220 \\
\hline \multirow{3}{*}{ S-bAFSA } & 10000 & 11539 & 11533 & 11537.92 & 11538 & 0.7239 \\
& 30000 & 11538 & 11538 & 11538 & 11538 & 0 \\
& 50000 & 11543 & 11538 & 11538.10 & 11538 & 0.7071 \\
\hline \multirow{3}{*}{ CGMA } & 10000 & 11543 & 11530 & 11538.36 & 11538 & 2.6936 \\
& 30000 & 11543 & 11537 & 11540.04 & 11540 & 2.2220 \\
& 50000 & 11543 & 11537 & 11541.16 & 11542 & 2.1887 \\
\hline
\end{tabular}

Table 11. The results of the test problems F62 with different maxFEs

\begin{tabular}{|c|c|c|c|c|c|c|}
\hline Algorithm & maxFEs & Best & Worst & Mean & Median & Std \\
\hline \multirow{3}{*}{ BPSO } & 10000 & 13252 & 12450 & 12863.14 & 12863 & 165.8154 \\
& 30000 & 13537 & 12465 & 12938.54 & 12931.50 & 194.0300 \\
& 50000 & 13923 & 13423 & 13701.98 & 13685 & 110.5425 \\
\hline \multirow{3}{*}{ MBPSO } & 10000 & 13814 & 12910 & 13448.32 & 13431.50 & 158.6587 \\
& 30000 & 13720 & 13136 & 13478.86 & 13482 & 120.6970 \\
& 50000 & 13786 & 13518 & 13498.40 & 13520 & 139.2587 \\
\hline \multirow{3}{*}{ NGHS } & 10000 & 11550 & 9407 & 10816.60 & 10893 & 446.7960 \\
& 30000 & 11920 & 9801 & 10873.92 & 10936 & 518.6333 \\
& 50000 & 11558 & 9768 & 10920.60 & 13510 & 507.6646 \\
\hline \multirow{3}{*}{ DGHS } & 10000 & 14319 & 14302 & 14312.26 & 14312.50 & 5.1142 \\
& 30000 & 14322 & 14316 & 14318.78 & 14319 & 1.7296 \\
& 50000 & 14325 & 14307 & 14320.44 & 14321.50 & 3.6821 \\
\hline \multirow{2}{*}{ S-bAFSA } & 10000 & 14325 & 14307 & 14320.60 & 14320 & 3.8439 \\
& 30000 & 14325 & 14311 & 14321.04 & 14321 & 3.2132 \\
\hline
\end{tabular}




\begin{tabular}{|c|c|c|c|c|c|c|}
\hline & 50000 & 14325 & 14315 & 14322.02 & 14322 & 2.5911 \\
\hline \multirow{3}{*}{ CGMA } & 10000 & 14325 & 14303 & 14316.98 & 14318 & 5.6442 \\
& 30000 & 14325 & 14307 & 14320.44 & 14321.50 & 3.6821 \\
& 50000 & 14325 & 14312 & 14321.50 & 14323 & 3.3335 \\
\hline
\end{tabular}

Table 12. The results of the test problems F63 with different maxFEs

\begin{tabular}{|c|c|c|c|c|c|c|}
\hline Algorithm & maxFEs & Best & Worst & Mean & Median & Std \\
\hline \multirow{3}{*}{ BPSO } & 10000 & 15613 & 14582 & 15086.96 & 15085.50 & 218.9724 \\
& 30000 & 15770 & 14636 & 15199.24 & 15199 & 212.0181 \\
& 50000 & 16529 & 15848 & 15086.96 & 15085.50 & 218.9724 \\
\hline \multirow{3}{*}{ MBPSO } & 10000 & 16313 & 15643 & 15920.50 & 15897 & 173.8603 \\
& 30000 & 16569 & 15784 & 16146.80 & 16127 & 170.7027 \\
& 50000 & 16836 & 15826 & 16187.52 & 16166.50 & 189.3395 \\
\hline \multirow{3}{*}{ NGHS } & 10000 & 13999 & 12111 & 13202.14 & 13255 & 376.7673 \\
& 30000 & 14207 & 12696 & 133.9190 & 13331 & 382.5406 \\
& 50000 & 14041 & 12692 & 134.7992 & 13510 & 327.4805 \\
\hline \multirow{3}{*}{ DGHS } & 10000 & 17708 & 17650 & 17688.06 & 17690 & 16.3138 \\
& 30000 & 17712 & 17706 & 17708.38 & 17708 & 1.7944 \\
& 50000 & 17718 & 17704 & 17713.78 & 17715 & 3.4004 \\
\hline \multirow{3}{*}{ S-bAFSA } & 10000 & 17718 & 17686 & 17707.22 & 17706 & 5.5633 \\
& 30000 & 17718 & 17706 & 17711.04 & 17711 & 3.8859 \\
& 50000 & 17718 & 17706 & 17712.54 & 17713 & 3.5869 \\
\hline \multirow{3}{*}{ CGMA } & 10000 & 17715 & 17664 & 17698.38 & 17701 & 12.2072 \\
& 30000 & 17718 & 17704 & 17713.78 & 17715 & 3.4004 \\
& 50000 & 17718 & 17704 & 17715.28 & 17716 & 4.4109 \\
\hline
\end{tabular}

Table 13. The results of the test problems F64 with different maxFEs

\begin{tabular}{|c|c|c|c|c|c|c|}
\hline Algorithm & maxFEs & Best & Worst & Mean & Median & Std \\
\hline \multirow{3}{*}{ BPSO } & 10000 & 36768 & 35237 & 35933.88 & 35909.50 & 363.5089 \\
& 30000 & 37070 & 35732 & 36374.58 & 36317 & 279.3405 \\
& 50000 & 37854 & 36525 & 37305.26 & 37322.50 & 301.1055 \\
\hline \multirow{3}{*}{ MBPSO } & 10000 & 37623 & 36441 & 36978.36 & 36934 & 301.6178 \\
& 30000 & 38124 & 37131 & 37742.14 & 37769 & 245.5838 \\
& 50000 & 38261 & 37194 & 37794.84 & 37832.50 & 262.4363 \\
\hline \multirow{3}{*}{ NGHS } & 10000 & 31409 & 28445 & 30133.76 & 30187 & 619.9649 \\
& 30000 & 31276 & 28760 & 30072.04 & 30153.50 & 574.1515 \\
& 50000 & 31666 & 29140 & 30223.06 & 30197 & 459.6279 \\
\hline \multirow{3}{*}{ DGHS } & 10000 & 39994 & 39566 & 39901.06 & 39906 & 75.1245 \\
& 30000 & 40211 & 39999 & 40100.80 & 40111 & 63.9793 \\
& 50000 & 40211 & 39999 & 40122.10 & 40111 & 78.6790 \\
\hline \multirow{3}{*}{ S-bAFSA } & 10000 & 40215 & 40211 & 40211.82 & 40211 & 1.5345 \\
& 30000 & 40215 & 40211 & 40212.56 & 40212 & 1.6801 \\
& 50000 & 40215 & 40211 & 40213.28 & 40214 & 1.7147 \\
\hline
\end{tabular}




\begin{tabular}{|c|c|c|c|c|cc|c|}
\hline & 10000 & 40211 & 39999 & 40122.10 & 40111 & 78.6790 \\
CGMA & 30000 & 40211 & 40160 & 40187.08 & 40188 & 14.0231 \\
& 50000 & 40211 & 40201 & 40209.64 & 40211 & 3.0288 \\
\hline
\end{tabular}

Table 14. The results of the test problems F65 with different maxFEs

\begin{tabular}{|c|c|c|c|c|c|c|}
\hline Algorithm & maxFEs & Best & Worst & Mean & Median & Std \\
\hline \multirow{3}{*}{ BPSO } & 10000 & 64551 & 63224 & 63979.54 & 63992 & 280.4874 \\
\hline & 30000 & 64915 & 63250 & 64118.12 & 64176.50 & 316.8198 \\
\hline & 50000 & 64959 & 63817 & 64428.70 & 64410.50 & 274.0309 \\
\hline \multirow{3}{*}{ MBPSO } & 10000 & 65013 & 64293 & 64706.54 & 64723 & 180.4052 \\
\hline & 30000 & 65963 & 65038 & 65536.22 & 65524 & 20.68221 \\
\hline & 50000 & 66102 & 65004 & 65598.82 & 65579.50 & 224.0022 \\
\hline \multirow{3}{*}{ NGHS } & 10000 & 63391 & 61792 & 62264.76 & 62157 & 350.3046 \\
\hline & 30000 & 63164 & 61863 & 62472.68 & 62496 & 317.9862 \\
\hline & 50000 & 63361 & 61531 & & 62542.50 & \\
\hline \multirow{3}{*}{ DGHS } & 10000 & 67426 & 67391 & 67404.58 & 67401 & 10.1521 \\
\hline & 30000 & 67426 & 67401 & 67416.58 & 67417 & 5.1233 \\
\hline & 50000 & 67427 & 67401 & 67418.76 & 67419.50 & 5.6625 \\
\hline \multirow{3}{*}{ S-bAFSA } & 10000 & 67428 & 67405 & $67415 . .28$ & 67416 & 5.6026 \\
\hline & 30000 & 67426 & 67410 & 67418.96 & 67419 & 3.5746 \\
\hline & 50000 & & & 67420.54 & 67420 & 3.0319 \\
\hline \multirow{3}{*}{ CGMA } & 10000 & 67424 & 67412 & 67417.90 & 67418.50 & 2.4265 \\
\hline & 30000 & 67427 & 67401 & 67418.76 & 67419.50 & 5.6625 \\
\hline & 50000 & 67428 & 67412 & 67425.10 & 67426 & 3.1960 \\
\hline
\end{tabular}

Table 15. The results of the test problems F66 with different maxFEs

\begin{tabular}{|c|c|c|c|c|c|c|}
\hline Algorithm & maxFEs & Best & Worst & Mean & Median & Std \\
\hline \multirow{3}{*}{ BPSO } & 10000 & 85371 & 84280 & 84924.70 & 84939 & 214.7547 \\
\hline & 30000 & 85815 & 84872 & 85319.46 & 85349.50 & 257.0525 \\
\hline & 50000 & 85080 & 85498 & 85833.02 & 85823 & 127.2609 \\
\hline \multirow{3}{*}{ MBPSO } & 10000 & 85528 & 84693 & 85171.40 & 85198.50 & 204.7106 \\
\hline & 30000 & 86342 & 85505 & 85945.10 & 85943.50 & 175.2100 \\
\hline & 50000 & 86310 & 85670 & 86029.50 & 86029 & 132.3053 \\
\hline \multirow{3}{*}{ NGHS } & 10000 & 85366 & 84384 & 84924.40 & 84938 & 382.8406 \\
\hline & 30000 & 85541 & 84567 & 85125.42 & 85129 & 240.0429 \\
\hline & 50000 & 85512 & 84357 & 85170.88 & 85200.50 & 265.7676 \\
\hline \multirow{3}{*}{ DGHS } & 10000 & 87164 & 87162 & 87163.82 & 87164 & 0.4375 \\
\hline & 30000 & 87167 & 87160 & 87163.34 & 87164 & 1.5066 \\
\hline & 50000 & 87167 & 87151 & 87165.18 & 87167 & 4.9844 \\
\hline \multirow{3}{*}{ S-bAFSA } & 10000 & 87166 & 87158 & 87161.16 & 87161 & 2.1887 \\
\hline & 30000 & 87167 & 87158 & 87162.22 & 87163 & 2.3672 \\
\hline & 50000 & 87166 & 87160 & 87163.44 & 87164 & 1.2803 \\
\hline CGMA & 10000 & 87167 & 87149 & 87164.46 & 87167 & 5.6648 \\
\hline
\end{tabular}




\begin{tabular}{|l|l|l|l|l|l|l|}
\hline & 30000 & 87167 & 87151 & 87165.18 & 87167 & 4.9844 \\
& 50000 & 87167 & 87149 & 87166.34 & 87167 & 3.1919 \\
\hline
\end{tabular}

Table 16. The results of the test problems F67 with different maxFEs

\begin{tabular}{|c|c|c|c|c|c|c|}
\hline Algorithm & maxFEs & Best & Worst & Mean & Median & Std \\
\hline \multirow{3}{*}{ BPSO } & 10000 & 97444 & 96054 & 96704.26 & 96729.50 & 346.4074 \\
\hline & 30000 & 97945 & 96077 & 97187.82 & 97243 & 391.1740 \\
\hline & 50000 & 98895 & 97928 & 98463.44 & 98492.50 & 231.5866 \\
\hline \multirow{3}{*}{ MBPSO } & 10000 & 99337 & 97971 & 98614.96 & 98647.50 & 289.4599 \\
\hline & 30000 & 100754 & 100081 & 100388.91 & 100366 & 185.7568 \\
\hline & 50000 & 101142 & 100132 & 100701.62 & 100720.50 & 224.9488 \\
\hline \multirow{3}{*}{ NGHS } & 10000 & 97394 & 95681 & 96626.90 & 96648.50 & 382.8406 \\
\hline & 30000 & 97664 & 95456 & 96847.78 & 96902.50 & 414.1766 \\
\hline & 50000 & 98032 & 95860 & 97064.34 & 97011 & 383.1199 \\
\hline \multirow{3}{*}{ DGHS } & 10000 & 103146 & 103123 & 103136.92 & 103137.50 & 5.9551 \\
\hline & 30000 & 103147 & 103140 & 103145.54 & 103146 & 1.8321 \\
\hline & 50000 & 103152 & 103116 & 103143.58 & 103144 & 6.0836 \\
\hline \multirow{3}{*}{ S-bAFSA } & 10000 & 103151 & 103113 & 103137.24 & 103142 & 13.2396 \\
\hline & 30000 & 103152 & 103119 & 103142.36 & 103147.50 & 10.7737 \\
\hline & 50000 & 103152 & 103119 & 103144.52 & 103151 & 10.2405 \\
\hline \multirow{3}{*}{ CGMA } & 10000 & 103147 & 103134 & 103142.90 & 103144 & 4.0489 \\
\hline & 30000 & 103152 & 103116 & 103143.61 & 103144 & 6.0836 \\
\hline & 50000 & 103151 & 103133 & 103145.70 & 103147 & 5.4595 \\
\hline
\end{tabular}

The tables 9 to 16 record the results of all the algorithms with different maxFEs for the test problems F60 to F67. The mean values of CGMA are better than that of other algorithms for most test problems with the same maxFEs; even the worst values are better than that of BPSO, MBPSO and NGHS on all the test problems except F60. For most test problems, the mean values of all the algorithms obviously present a positive relation with the increase in maxFEs. However, the mean values of NGHS may fall for some test problems, such as F60. In particular, the growth rates of mean values are great for BPSO and MBPSO.

The most significant characteristic of MA compared to other algorithms is the somersault process, which effectively avoids monkeys running into the local optimal solutions. However, the somersault process may lose efficacy, and monkeys may fall into the local optimal solutions after many iterations. To overcome this problem, this paper improves the somersault process and introduces a control parameter.

For the MBPSO algorithm, the position update equation is redesigned [6]. MBPSO provides better exploration than BPSO in solving the 0-1 knapsack problem. However, it is evident that MBPSO is highly dependent on the constant maximum velocity [6]. Sometimes, it is difficult to set a proper maximum velocity in solving different knapsack problems. For BPSO, it is easy to fall into local solutions and the stability of BPSO is poor compared with CGMA. 
For the DGHS algorithm, the two-phase repair operator [3] is introduced to strengthen its local search ability. It is observed that the standard deviation of DGHS is small on all test problems; that is, it has strong stability for solving the 0-1 knapsack problem. For NGHS, the global exploration ability is obviously inferior to DGHS. It is easy to fall into the local optima.

For the S-bAFSA algorithm [7], the random heuristic drop_item procedure is used to make the points feasible, and the heuristic add_item is also implemented to improve the quality of the solutions. S-bAFSA has better performance than CGMA in solving strongly correlated problems, such as F48, F49, F52, F54, F58, F59. In addition, S-bAFSA presents strong robustness.

\section{2) The robustness test of CGMA}

As observed in table 9, CGMA can obtain the same value 7481 in all runs on the test problem F60 with maxFEs $=50000$. It is also worth mentioning that the standard deviation obtained by CGMA is very small on each test problem. So, CGMA has strong robustness. To test the robustness of CGMA, here, we generate 5 instances with the size $n=100,120,130,140,150$, and the maximum capacity of knapsack $V=1100,1200,1230,1270,1300$; the results of CGMA are recorded in the table 10 for 50 independent runs.

Table 17. The results of CGMA for the different sizes

\begin{tabular}{|c|c|c|c|c|c|c|}
\hline Problem & Dimension & Best & Worst & Mean & Median & Std \\
\hline F68 & 100 & 6654 & 6654 & 6654 & 6654 & 0 \\
\hline F69 & 120 & 7933 & 7933 & 7933 & 7933 & 0 \\
\hline F70 & 130 & 8554 & 8554 & 8554 & 8554 & 0 \\
\hline F71 & 140 & 8358 & 8355 & 8357.58 & 8358 & 0.9278 \\
\hline F72 & 150 & 8704 & 8702 & 8703.45 & 8704 & 0.8256 \\
\hline
\end{tabular}

As observed in table 17, when the size of the problem is less than or equal to 130, CGMA can obtain the same global optimal value for 50 runs. When the size is greater than or equal to140, the same value cannot be found for every experiment. Here, we generate 5 different instances with the same size of 130 to test; table 18 shows the results. For the five test problems, CGMA can obtain the same value except the problem F56. The results show that CGMA has strong robustness and stability for solving the 0-1 Knapsack problem.

Table 18. The results of CGMA for 5 different instances with the same size

\begin{tabular}{|c|c|c|c|c|c|c|}
\hline Problem & Dimension & Best & Worst & Mean & Median & Std \\
\hline F73 & 130 & 8924 & 8924 & 8924 & 8924 & 0 \\
\hline F74 & 130 & 8308 & 8308 & 8308 & 8308 & 0 \\
\hline F75 & 130 & 8551 & 8551 & 8551 & 8551 & 0 \\
\hline F76 & 130 & 8238 & 8235 & 8238 & 8237.88 & 0.4798 \\
\hline F77 & 130 & 8028 & 8028 & 8028 & 8028 & 0 \\
\hline
\end{tabular}




\section{3) The effect of different climb numbers on the performance of MA and CGMA}

For the original MA, the experiment demonstrating the precision of the solution is determined by the climb step and the climb number for the optimization problem [8]. For the knapsack problem, the climb step is set to 1 , so the precision of the solution is determined by the climb number. The larger the climb number, the better is the value. To test the impact of the climb number, the maxFE is set to 50000. The population size is set to 50. Table 19 shows the results for MA with different climb numbers $N_{c}$ for the test problems F36 to F39. As observed from the results, when the climb number is 50, the mean value of MA is better, and the standard deviation is smaller. So, for MA, we can improve the quality of the solution by increasing the climb number. However, the precision of the solution is not impacted by different climb numbers due to the optimization of the greedy algorithm for CGMA.

Table 19. The effect of climb number on the performance of MA

\begin{tabular}{|c|c|c|c|c|c|c|}
\hline \multirow{4}{*}{ Problem } & Climb number & Best & Worst & Mean & Median & Std \\
\hline \multirow{3}{*}{ F36 } & 5 & 3019 & 2958 & 2979.94 & 2976.50 & 14.8945 \\
\cline { 2 - 7 } & 20 & 3022 & 2969 & 2998 & 2998.50 & 11.6128 \\
\cline { 2 - 7 } & 50 & 3103 & 2997 & 3059.71 & 3083 & 38.6101 \\
\cline { 2 - 7 } & 5 & 4691 & 4374 & 4488.61 & 4477.50 & 67.5480 \\
\hline \multirow{3}{*}{ F37 F38 } & 20 & 4712 & 4465 & 4552.31 & 4541 & 52.8072 \\
\cline { 2 - 7 } & 50 & 4749 & 4523 & 4603.82 & 4603 & 46.9814 \\
\cline { 2 - 7 } & 5 & 4564 & 4263 & 4386.14 & 4383.50 & 68.1235 \\
\hline \multirow{3}{*}{ F39 } & 50 & 4619 & 4349 & 4442.90 & 4431.50 & 64.4660 \\
\hline
\end{tabular}

\section{Conclusions}

This paper proposed a binary monkey algorithm for solving 0-1 knapsack problems. The algorithm used the greedy algorithm to correct the infeasible solutions and to improve the feasibility, introduced the cooperation process to speed up the convergence rate, modified the somersault process by randomly choosing one monkey as the pivot of another to avoid falling into the local optimal solutions, and reinitialized the population if the global optimal solution was not improved after a predetermined number of generations. The experiments show that the proposed CGMA algorithm has strong advantages in solving 0-1 knapsack problems for testing fixed and random problems and small- and large-scale problems. CGMA can be an efficient alternative for solving the 0-1 knapsack problem.

\section{Acknowledgments}

This work is supported by National Science Foundation of China under Grants No. 61165015, 61463007,61563008 . 


\section{References}

[1] Dantzig G B. Discrete variable extremum problems. Operations Research 5: 266-77. Nature, 1957, 183(4): 189-202.

[2] Zou D X, Gao L Q, Li S, et al. Solving 0-1 knapsack problem by a novel global harmony search algorithm, Applied Soft Computing. 2011, 11(2): 1556-1564.

[3] Xiang W L, An M Q, Li Y Z, et al. A Novel Discrete Global-Best Harmony Search Algorithm for Solving 0-1 Knapsack Problems. Discrete Dynamics in Nature and Society, 2014(2014). Article ID 573731, 12 pages, doi.org/10.1155/2014/573731.

[4] Changdar C, Mahapatra G S, Pal R K. An Ant colony optimization approach for binary knapsack problem under fuzziness. Applied Mathematics and Computation, 2013, 223: 243-253.

[5] Kennedy J, Eberhart R C. Swarm intelligence. Morgan Kaufmann Publishers, 2001.

[6] Bansal J C, Deep K. A modified binary particle swarm optimization for knapsack problems. Applied Mathematics and Computation, 2012, 218(22): 11042-11061.

[7] Azad M A K, Rocha A M A C, Fernandes E M G P. A simplified binary artificial fish swarm algorithm for 0-1quadratic knapsack problems. Journal of Computational and Applied Mathematics, 2014, 259: 897-904.

[8] Glover F. Advanced greedy algorithms and surrogate constraint methods for linear and quadratic knapsack and covering problems. European Journal of Operational Research, 2013, 230(2): 212-225.

[9] Sitarz S. Multiple criteria dynamic programming and multiple knapsack problem. Applied Mathematics and Computation, 2014, 228: 598-605.

[10] Gao J, He G, Liang R, et al. A quantum-inspired artificial immune system for the multiobjective 0-1 knapsack problem. Applied Mathematics and Computation, 2014, 230: 120-137.

[11] García-Martínez C, Rodriguez F J, Lozano M. Tabu-enhanced iterated greedy algorithm: A case study in the quadratic multiple knapsack problem. European Journal of Operational Research, 2014, 232(3): 454-463.

[12] Baykasolu A, Ozsoydan F B. An improved firefly algorithm for solving dynamic multidimensional knapsack problems. Expert Systems with Applications, 2014, 41(8): 3712-3725.

[13] Hifi M, Wu L. New upper bounds and exact methods for the knapsack sharing problem. Applied Mathematics and Computation, 2014, 227: 518-530.

[14] Levin A, Vainer A. Adaptivity in the stochastic blackjack knapsack problem. Theoretical Computer Science, 2014, 516: 121-126.

[15] Zhao J Y. Nonlinear Reductive Dimension Approximate Algorithm for 0-1 Knapsack Problem. Journal of Inner Mongolia Normal University (Natural Science Edition), 2007, 1: 005

[16] Liu A Z, Wang J Z, Han G D, et al. Improved simulated annealing algorithm solving for 0/1 knapsack problem. Intelligent Systems Design and Applications, 2006. ISDA'06. Sixth International Conference on. IEEE, 2006, 2: 1159-1164.

[17] Saraç T, Sipahioglu A. Generalized quadratic multiple knapsack problem and two solution approaches. Computers \& Operations Research, 2014, 43: 78-89.

[18] Lin F T. Solving the knapsack problem with imprecise weight coefficients using genetic algorithms. European Journal of Operational Research, 2008, 185(1): 133-145.

[19] Zhao R Q, Tang W S. Monkey algorithm for global numerical optimization. Journal of Uncertain Systems. 2008,2 (3):164-175.

[20] Chen X, Zhou Y Q. Hybrid Algorithm Based on Monkey Algorithm and Simple Method. Computer Science, 2013, 40(11): 2477-254.

[21] Wang J R, Yu Y X, Zeng Y. Discrete monkey algorithm and its application in transmission network expansion planning. 2010 IEEE Power and Energy Society General Meeting, IEEE, 2010: 1-5. 
[22] Zhang J J, Zhang Y P, Sun J Z. Intrusion Detection Technology Based on Monkey Algorithm. Computer Engineering. 2011, 37(14): 131-133.

[23] Yi T H, Li H N, Zhang X D. A modified monkey algorithm for optimal sensor placement in structural health monitoring. Smart Materials and Structures. 2012, 21(10): 65-69.

[24] Zhao T, Xia Y, Zong M L. Optimization of Gas Filling Station Project Scheduling Problem Based on Monkey Algorithm. Value Engineering. 2010: 90-92.

[25] Chen X, Zhou Y Q, Luo Q F. A Hybrid Monkey Search Algorithm for Clustering Analysis. The Scientific World Journal. 2014, 2014. Article ID 938239, 16 pages. doi: 10.1155/2014/938239.

[26] Gao F, Cui G, Wu Z B, et al. Virus-evolutionary Particle Swarm Optimization Algorithm for Knapsack Problem. Journal of Harbn Institute of Technology. 2009, 41(6): 103-107.

[27] Spall J. Multivariate Stochastic Approximation using a Simultaneous Perturbation Gradient Approximation. IEEE Transactions on Automatic Control. 1992, 37: 332-341.

[28] Liu J Q, He Y C, Gu Qian Q. Solving knapsack problem based on discrete particle swarm optimization. Computer Engineering and Design, 2007, 29(13): 3189-3191.

[29] Lee C Y, Lee Z J, Su S F, A new approach for solving 0/1 knapsack problem, IEEE International Conference on Systems, Man, and Cybernetics October 8-11, Taipei, Taiwan, 2006, pp. 3138-3143.

[30] Chen K, Ma L. Artificial glowworm swarm optimization algorithm for 0-1 knapsack problem. Application Research of Computers. 2013, 30(4): 996-998.

[31] Feng Y, Wang G G, Feng Q J, et al. An Effective Hybrid Cuckoo Search Algorithm with Improved Shuffled Frog Leaping Algorithm for 0-1 Knapsack Problems. Computational intelligence and neuroscience, 2014, 2014 (2014), Article ID 857254, 17 pages. doi.org/10.1155/2014/857254.

[32] Shi H X. Solution to 0/1 knapsack problem based on improved ant colony algorithm. Information Acquisition, 2006 IEEE International Conference on. IEEE, 2006: 1062-1066. 